\title{
Enhancement in soft breakdown occurrence of ultra-thin gate oxides caused by photon effect in rapid thermal post-oxidation annealing
}

\author{
Chia-Hong Huang, Jenn-Gwo Hwu * \\ Department of Electrical Engineering, National Taiwan University, Room 446, Taipei, Taiwan, ROC
}

Received 5 January 2000; received in revised form 6 April 2000; accepted 8 May 2000

\begin{abstract}
Soft breakdown properties affected by photon energy during rapid thermal post-oxidation annealing (POA) of ultrathin gate oxide are investigated. Generally, breakdown can be classified into normal hard breakdown (HBD) and soft breakdown (SBD). It was found that the occurrence of HBD and SBD depends on the process and stress field. Samples with front and back sides illuminated by a tungsten-halogen lamp during rapid thermal POA were examined. We find that front side illuminated oxides show easier SBD than back side ones. These results indicate that the photon energy may not only enhance the strength of $\mathrm{Si}-\mathrm{O}$ bonds to suppress the lateral propagation of breakdown spots, but also to localize defects to form a conductive path of the leakage current. The understanding of the photon effect in rapid thermal POA is useful for controlling the electrical properties of ultra-thin gate oxides. (c) 2000 Elsevier Science Ltd. All rights reserved.
\end{abstract}

Keywords: Soft breakdown; Post-oxidation annealing; Hard breakdown

\section{Introduction}

The ultra-thin gate oxide reliability is one of the most important issues in future ultra-large scale integration (ULSI). From the Semiconductor Industry Association's National Technology Roadmap, it is known that for a semiconductor beyond $0.13 \mu \mathrm{m}$ technology, the gate oxide thickness will be less than $3 \mathrm{~nm}$. Recent reports have shown that the wear-out behavior of these ultra-thin gate oxides can be classified into two modes under high electrical field stress [1,2]. One is hard breakdown (HBD) and the other is soft breakdown (SBD). The latter is also called quasi-breakdown [3] or partial breakdown [4]. It was found that the stressinduced leakage current (SILC) increases sharply for ultra-thin gate oxides after electrical stress $[1-3,5,6]$. It

\footnotetext{
${ }^{*}$ Corresponding author. Tel.: +886-2-23635251, ext.: 446; fax: +886-2-23671909.

E-mail address: hwu@cc.ee.ntu.edu.tw (J.-G. Hwu).
}

has been reported that SBD is defined as oxide breakdown without lateral propagation of the breakdown spot due to thermal damage [1]. Therefore, when SBD occurs, the oxide must be considered as a failure oxide. Consequently, when SBD is not taken into account in time-dependent dielectric breakdown (TDDB) tests, the ultra-thin gate oxide reliability is overestimated [1]. Nevertheless, some reports state that even though SBD occurs, a device still remains functioning, but SBD can cause noise in the device operation [7]. Therefore, circuit performance is influenced by the SBD characteristics of ultra-thin gate oxide. A fabrication process of ultra-thin gate oxide with less possibility for SBD to occur is of interest.

A recent report shows that optical radiation during rapid thermal annealing (RTA) assists in the migration of boron through the oxide, and at the peak blackbody temperature of a tungsten-halogen lamp in an RTA system, significant numbers of electron-hole pairs are generated in $\mathrm{SiO}_{2}$ [8]. In rapid thermal processing (RTP) based on incoherent light as the source of energy, the 
generated microscopic defects in oxides are different from that in a conventional furnace. Moreover, the microscopic defects are much larger for RTP with back heating compared to that with front heating [9]. Here, the effect of front- and back-side heated post-oxidation annealing (POA) on ultra-thin gate oxide in RTP is studied. We find that the oxide breakdown property is dependent on the photon effect in POA. The characterization of oxide breakdown is examined by the occurrence frequency of SBD and HBD. An enhancement of SBD occurrence frequency is clearly observed for the front-side heated POA samples. Possible reasons for the observation are given.

\section{Experimental}

The devices used in this study are metal-oxide-semiconductor (MOS) capacitor structures with an aluminum gate electrode fabricated on a $(100)$ p-type $\mathrm{Si}$ substrate. The resistivity of the silicon substrate used in this study is $1-5 \Omega \mathrm{cm}$. The gate oxide has been grown by rapid thermal oxidation (RTO) at $800^{\circ} \mathrm{C}$ for $35 \mathrm{~s}$, followed by front-side or back-side heating POA in $\mathrm{N}_{2}$ ambience, i.e., FPOA and BPOA, respectively, at $850^{\circ} \mathrm{C}$, $900^{\circ} \mathrm{C}$, and $960^{\circ} \mathrm{C}$. The schematic diagram of FPOA and BPOA apparatus is shown in Fig. 1. After POA, the aluminum gate electrode is fabricated by thermal evaporation. The gate electrode area is $150 \times 150 \mu \mathrm{m}^{2}$. Aluminum was also used as the backside contact. The oxide thickness ranging from 1.8 to $2.8 \mathrm{~nm}$ was determined by ellipsometry. The gate electrode was applied to a constant field stress, and the substrate was grounded. The applied high electrical field will introduce electron injection into the oxide. The time evolution of the gate current through the oxide was monitored automatically with an interval of $1 \mathrm{~s}$ during constant field stress. All measurements were carried out using a HP 4140B pA meter/dc voltage source. The field stress was interrupted periodically to measure the current-voltage $(I-V)$ char-

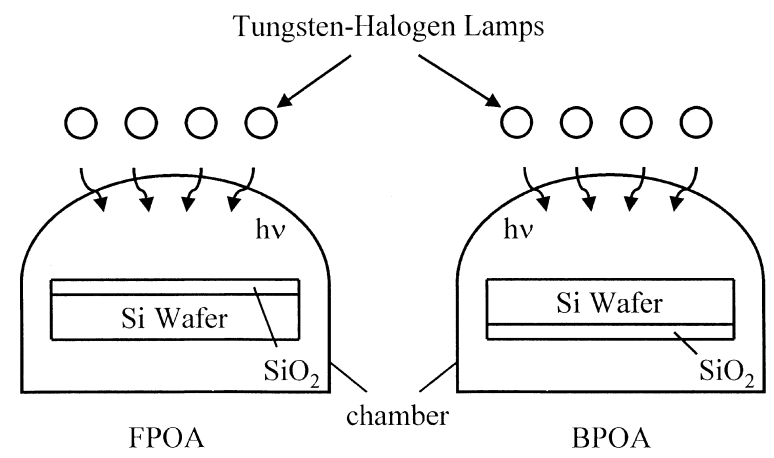

Fig. 1. Schematic diagram of FPOA and BPOA apparatus. acteristics of the degraded gate oxide. At least 30 devices were investigated for each process.

\section{Results and discussion}

When the ultra-thin gate oxide was subjected to electrical stress, it did not usually experience catastrophic breakdown, but it could be worn out through a localized conduction path [1]. Fig. 2(a) and (b) shows two typical SBD examples of the gate current (i.e., SBD1 and SBD2 defined later, respectively), versus stressing time for oxides with a thickness of $1.8 \mathrm{~nm}$ during -15 $\mathrm{MV} / \mathrm{cm}$ field stress. Due to the random distribution of defects and thickness nonuniformity, at least two kinds of SBD mechanisms are observed. For the first one, as shown in Fig. 2(a), the gate current is gradually decreasing when a constant field stress is applied, but the

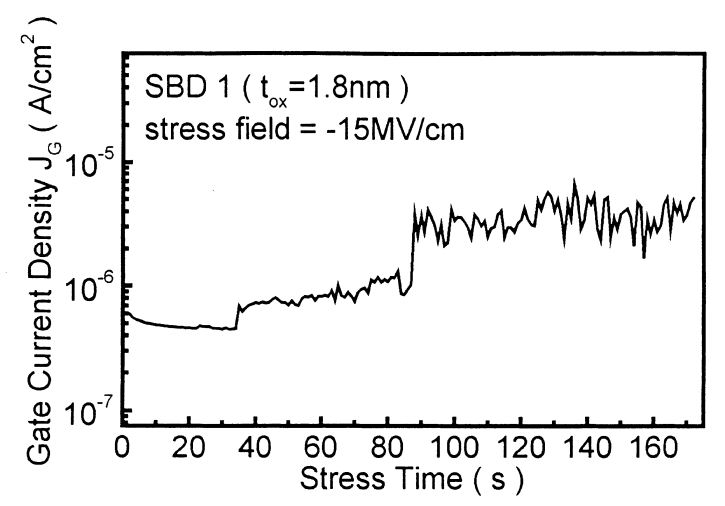

(a)

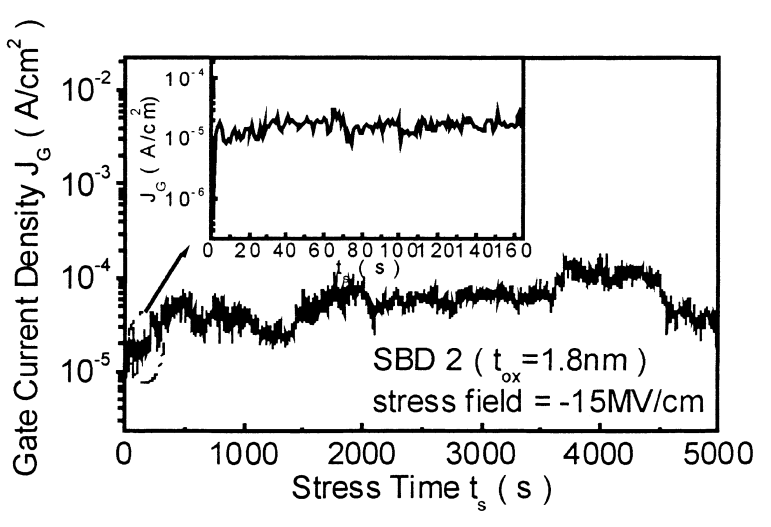

(b)

Fig. 2. Observed SBD current behavior during constant field stressing: (a) the gate current increases gradually as stress is applied for a certain time; (b) the gate current starts to fluctuate as soon as the stress is applied to gate electrode. 
amount of the decreasing current is small and is nearly constant. Therefore, it indicates small electron trapping in the oxide. After a short time, the gate current suddenly jumps and fluctuates. It is called SBD1. However, for the second one, it is observed that the gate current starts to fluctuate as soon as the stress is applied to the gate electrode, and HBD is not observed after a long stressing time. It is called SBD2 as shown in Fig. 2(b). They are both included in the SBD consideration in this study. Once SBD occurred and the gate current varied until destructive breakdown. However, it is difficult to observe HBD after an SBD event occurring in an oxide in the ultra-thin thickness $(2 \mathrm{~nm})$ regime [7]. So, in this study, we only deal with the first breakdown event in HBD characterization. That is to say that for the HBD samples, no SBD event occurs before HBD. It is noted that although the constant high field $(-15 \mathrm{MV} / \mathrm{cm})$ is applied to the oxide, the voltage across the oxide is relatively low so that a transition from direct tunneling to $F-N$ tunneling does not occur. Therefore, as the constant field stress is applied, direct tunneling is dominant. Moreover, the oxide thickness is less than $3 \mathrm{~nm}$ so that SILC is not dominant, since it is smaller than the direct tunneling current $[11,12]$.

Both FPOA and BPOA samples exhibit SBD1 and SBD2 behavior as mentioned above. Fluctuations in gate currents for both cases of FPOA and BPOA during constant field stress are indistinguishable. Furthermore, for FPOA and BPOA samples, it was found that their current-voltage $(I-V)$ characteristics after SBD are noisier, as shown in Fig. 3. Therefore, once SBD occurs, the $I-V$ characteristics does not change abruptly, but becomes noisier [7]. This result is similar to Weir's observation [7], but is different from the behavior of the thicker oxide $[1,2,6]$. So, we try to make use of breakdown statistics to distinguish the characteristics of FPOA and BPOA. The result of breakdown statistics is presented later. It is generally proposed that this noise is

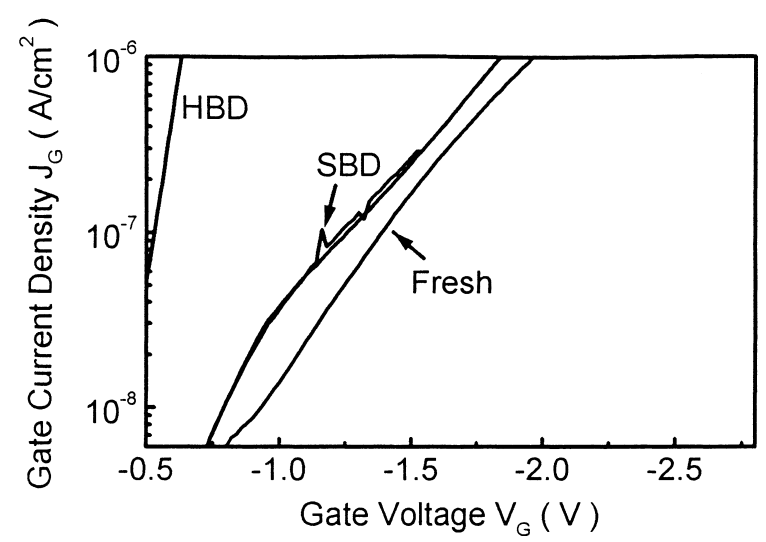

Fig. 3. The current-voltage characteristics of samples before and after the occurrence of SBD and HBD. mainly due to trap-assisted conduction through a small area of the capacitor which exists in almost all the ultrathin oxides $[7,13,14]$. It has been proposed that the percolation path is formed in the oxide at SBD, and the fluctuation observed in ultra-thin gate oxides after SBD is due to the trapping-detrapping of charges in the percolation cluster [15]. Any change of the defect distribution in the percolation path should result in the different fluctuation of gate current and breakdown behavior. However, the fluctuation of gate current is difficult to characterize. So, breakdown statistics is a possible approach to examine the property of FPOA and BPOA as discussed in the study.

In the TDDB test, we find that the time to hard breakdown of FPOA samples is longer than that of BPOA ones as shown in Fig. 4(a). It is noted that the SBD samples are not included in Fig. 4(a). Here, the cumulative failure value $F$ is obtained from the ratio of the cumulative HBD samples number to the total HBD samples number. This indicates that photon energy should enhance the strength of $\mathrm{Si}-\mathrm{O}$ bonds during POA

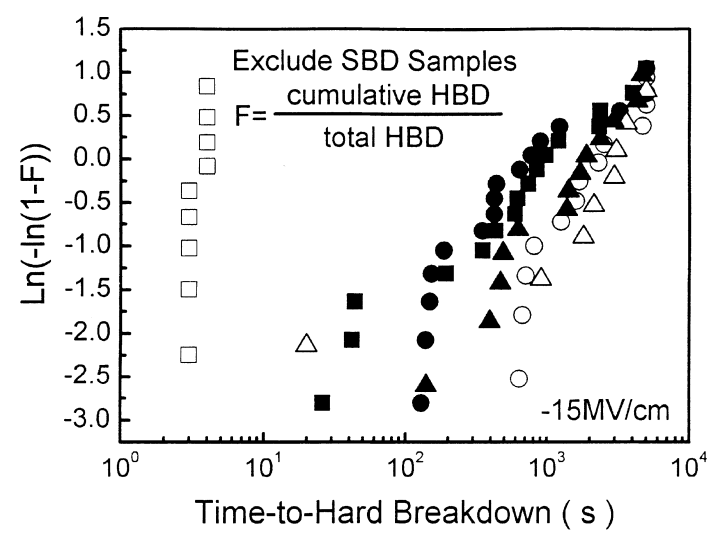

(a)

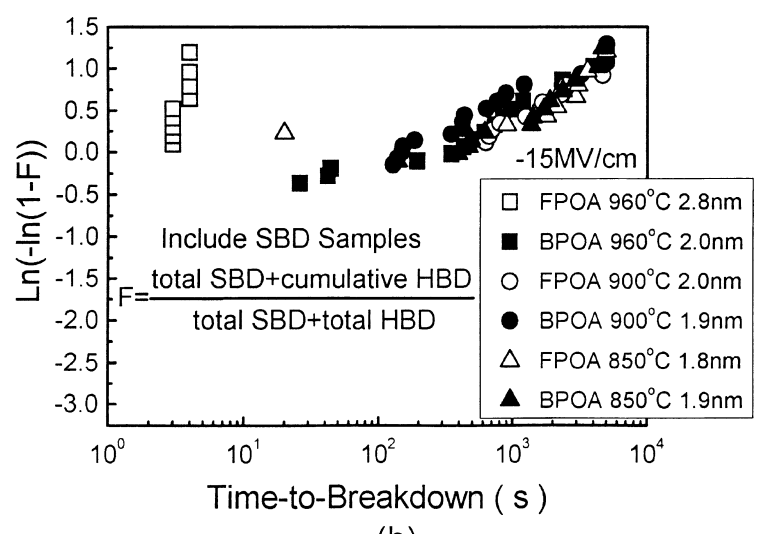

(b)

Fig. 4. Statistical distribution of the time-to-breakdown $\left(t_{\mathrm{BD}}\right)$ for samples (a) excluding capacitors with SBD occurrence and (b) including total dielectric breakdown of SBD and HBD. 
processing [8-10]. The primary difference in the two POA processes originates from the existence of special additional photon energy absorbed by oxide in FPOA but not in BPOA. In the case of BPOA processing, only thermal conduction energy is considered in the annealing. Nevertheless, for the case of FPOA processing, photons near the UV region are available [10]. It is proposed that the photons provide available energy to break the strained bonds or defects in an oxide so that they are easier to be reconstructed during annealing. Consequently, the stronger $\mathrm{Si}-\mathrm{O}$ bonds should suppress the lateral propagation of the breakdown spot due to the thermal damage in the FPOA samples.

As a matter of fact, it is quite difficult to obtain the time to soft breakdown for the SBD2 sample, since the gate current fluctuates under constant field stress in the beginning of the test as shown in Fig. 2(b). SBD2 separated from SBD events is not available. However, the total SBD sample number is obtainable and is considered in the breakdown statistics in this study. The SBD information is not included in Fig. 4(a). Therefore, to decide on a more accurate distribution of the lifetime for ultra-thin gate oxides, we include HBD and SBD events in the time-to-breakdown measurement as shown in Fig. 4(b). Now, the cumulative failure value $F$ is obtained from the ratio of the sum of the total SBD samples number and the cumulative HBD samples number to the total SBD and HBD samples numbers. We find that in Fig. 4(b), both the distributions of FPOA and BPOA samples are very close. This indicates that although the lifetime of HBD for FPOA is longer, the number of these samples is few. For FPOA $960^{\circ} \mathrm{C}$ samples, their time-tobreakdown are short in comparison to the others. This may account for their thicker oxide, i.e., $2.8 \mathrm{~nm}$, which allows a larger probability for the occurrence of impact ionization under high field stress.

SBD is frequently occurring in FPOA and BPOA samples. However, it is interesting that the occurrence frequency of SBD for FPOA samples is different from that for the BPOA ones. Fig. 5 shows the SBD and HBD occurrence frequencies histogram for various POA samples stressed under $-15 \mathrm{MV} / \mathrm{cm}$. The SBD occurrence frequency of FPOA is generally larger than those of BPOA. This result may be caused by the photon effect on the weak spots. We have tried to explain this result by the proposed photon enhancement effect. This model is built-up based on whether the density of defects in the local area reaches a critical density that can cause SBD occurrence during POA or not. Moreover, trap density modulations can occur through thermally activated motion of defects in the oxide [16]. SBD occurrence can be enhanced in the exposure of photons during POA. Consequently, it is supposed that for BPOA samples, only thermal conduction energy can be used to reduce the traps. The distribution of traps is still wide that it causes SBD occurrence difficult. But for FPOA samples,

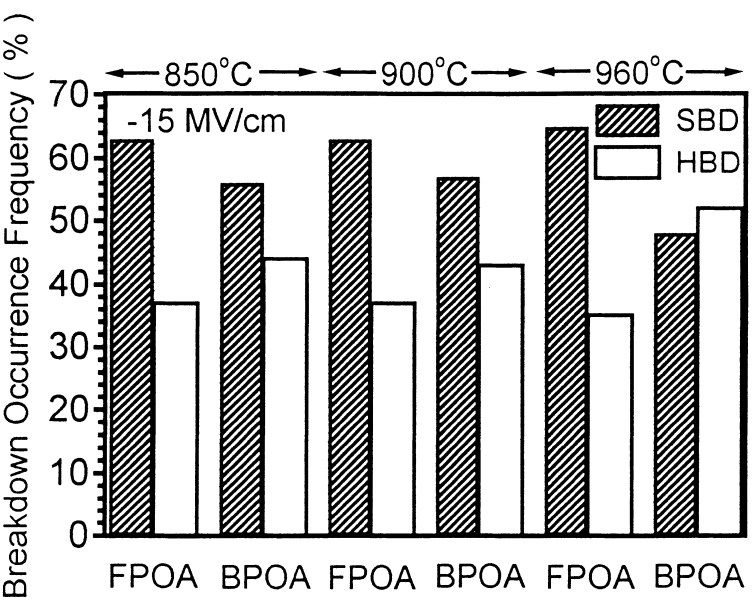

Fig. 5. SBD and HBD occurrence frequency histogram of various POA samples under $-15 \mathrm{MV} / \mathrm{cm}$ stress.

additional photon energy is helpful to reduce the traps. It leads to the local trap distribution with a density larger than the critical one that may cause an easy occurrence of SBD.

Fig. 6 shows the dependence of SBD occurrence frequency on stress field for FPOA and BPOA samples. It is found that the SBD occurrence frequency of BPOA decreases with the applied field. But for FPOA, the frequency saturates with applied field. The BPOA behavior is similar to that observed in the existing literature [2]. When the stress field is increased, the thermal damage will be large in the oxide breakdown so that it enhances the easy occurrence of HBD. Therefore, the possibility of SBD occurrence is decreased with the stress field [1,2]. For FPOA samples, localized weak

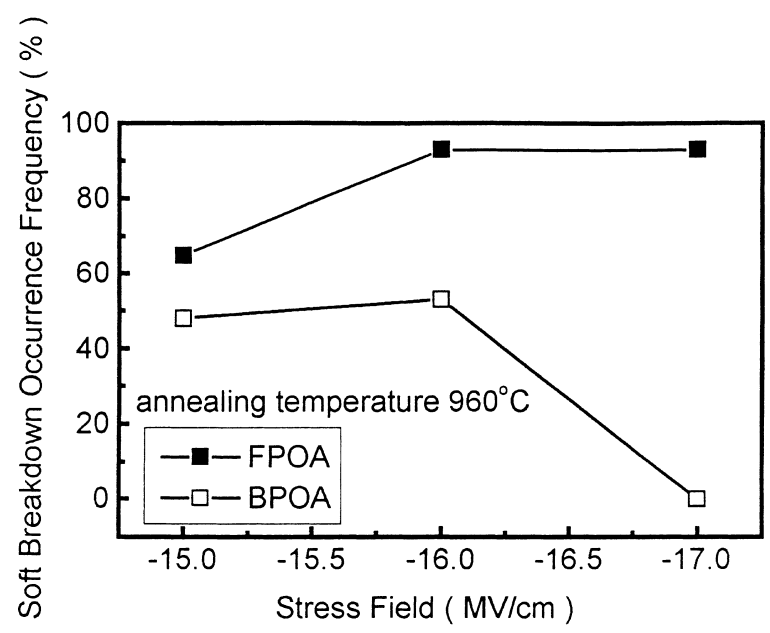

Fig. 6. SBD occurrence frequency of various POA samples with a POA temperature of $960^{\circ} \mathrm{C}$ versus stress field. 


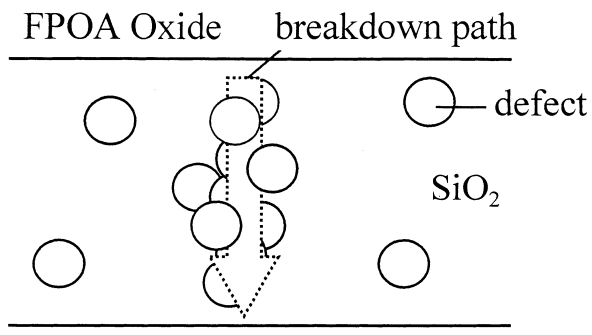

(a)

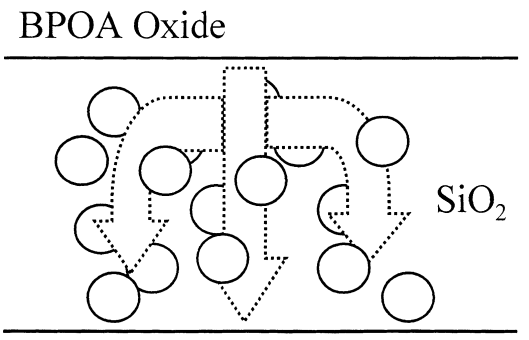

(b)

Fig. 7. Schematic diagram of the spatial distribution of defects under high field stress for samples after receiving (a) FPOA and (b) BPOA treatment.

defects enhanced by photon energy still cause a local conductive path under higher field stress. So, the SBD occurrence frequency of FPOA samples shows a saturation behavior as observed in Fig. 6 .

Fig. 7(a) and (b) gives the schematic diagram of the spatial distribution of defects and current conductive paths when breakdown occurs for FPOA and BPOA samples, respectively. The FPOA oxides receive photon irradiation and thermal energies from the front side. The defects are localized as shown in Fig. 7(a). But for BPOA oxides, only thermal conduction energy comes from the back side of wafer. Therefore, the defects are distributed much wider as shown in Fig. 7(b). These models can be used to explain the observations as mentioned above. The oxide properties can be affected by the rapid thermal POA treatment in view of the SBD characteristics.

\section{Conclusion}

We have observed that the SBD occurrence frequencies are different for FPOA and BPOA processes. It is attributed to the higher density of localized traps caused by the photon effect in POA processing. The distribution of defects is relatively wider for BPOA samples compared to FPOA. It is supposed that the photon energy does not only enhance the strength of $\mathrm{Si}-\mathrm{O}$ bonds to suppress the lateral propagation of the breakdown spot, but also to localize weak spots to form a conductive path for the leakage current. The above assumption was supported by the occurrence frequencies of SBD and HBD as observed in this work. It is useful to understand the photon effect on the electrical properties of ultra-thin oxides, especially for rapid thermal POA processing.

\section{Acknowledgements}

The authors want to thank the National Science Council of Republic of China for supporting this work under contract no. NSC 89-2215-E-002-010.

\section{References}

[1] Depas M, Nigam T, Heyns MM. Soft breakdown of ultrathin gate oxide layers. IEEE Trans Electron Dev 1996;43(9):1499-504.

[2] Tomita T, Utsunomiya H, Sakura T, Kamakura Y, Taniguchi K. A new soft breakdown model for thin thermal $\mathrm{SiO}_{2}$ films under constant current stress. IEEE Trans Electron Dev 1999;46(1):159-65.

[3] Lee SH, Cho BJ, Kim JC, Choi SH. Quasi-breakdown of ultrathin gate oxide under high field stress. IEDM Tech Dig 1994:605-8.

[4] Fu KY. Partial breakdown of the tunnel oxide in floating gate devices. Solid-State Electron 1997;41(5):774-7.

[5] Houssa M, Nigam T, Mertens PW, Heyns MM. Soft breakdown in ultrathin gate oxides: correlation with the percolation theory of nonlinear conductors. Appl Phys Lett 1998;73(4):514-6.

[6] Miranda E, Suñé J, Rodríguez R, Nafría M, Aymerich X. Switching behavior of the soft breakdown conduction characteristic in ultra-thin $(<5 \mathrm{~nm})$ oxide MOS capacitors. IRPS Tech Dig 1998:42-6.

[7] Weir BE, Silverman PJ, Monroe D, Krisch KS, Alam MA, Alers GB, Sorsch TW, Timp GL, Baumann F, Liu CT, Ma Y, Hwang D. Ultra-thin gate dielectrics: they break down, but do they fail? IEDM Tech Dig 1997:73-6.

[8] Fair RB. Anomalous B penetration through ultrathin gate oxides during rapid thermal annealing. IEEE Electron Dev Lett 1999;20(9):466-8.

[9] Singh R, Nimmagadda SV, Parihar V, Chen Y, Poole KF. Role of rapid photothermal processing in process integration. IEEE Trans Electron Dev 1998;45(3):643-54.

[10] Singh R, Radpour F, Chou P. Comparative study of dielectric formation by furnace and rapid isothermal processing. J Vac Sci Technol 1989;A7(3):1456-60.

[11] Chou AI, Lai K, Kumar K, Chowdhury P, Lee JC. Modeling of stress-induced leakage current in ultrathin oxides with the trap-assisted tunneling mechanism. Appl Phys Lett 1997;70(25):3407-9.

[12] Lai K, Chen W-M, Hao M-Y, Lee J. Turn-around effects of stress-induced leakage current of ultrathin $\mathrm{N}_{2} \mathrm{O}$ annealed oxides. Appl Phys Lett 1996;67(5):673-5. 
[13] Farmer KR, Saletti R, Buhrman RA. Current fluctuations and silicon oxide wear-out in metal-oxide-semiconductor tunnel diodes. Appl Phys Lett 1988;52(20): 1749-51.

[14] Farmer KR, Rogers CT, Buhrman RA. Localized-state interactions in metal-oxide-semiconductor tunnel diodes. Phys Rev Lett 1987;58(21):2255-8.
[15] Houssa M, Vandewalle N, Ausloos M, Mertens PW, Heyns MM. Analysis of the gate voltage fluctuations in ultra-thin gate oxides after soft breakdown. IEDM Tech Dig 1998:909-12.

[16] Alers GB, Krisch KS, Monroe D, Weir BE, Chang AM. Tunneling current noise in thin gate oxides. Appl Phys Lett 1996;69(19):2885-7. 